\title{
Glioblastoma multiforme: Molecular characterization and current treatment strategy (Review)
}

\author{
XIANG ZHANG $^{1 *}$, WEI ZHANG ${ }^{1 *}$, WEI-DONG CAO ${ }^{1}$, GANG CHENG $^{2}$ and YONG-QIANG ZHANG ${ }^{1}$ \\ ${ }^{1}$ Institute of Neurosurgery, and ${ }^{2}$ Department of Oncology, Xijing Hospital, \\ Fourth Military Medical University, Xi'an, Shaanxi, P.R. China
}

Received July 4, 2011; Accepted August 23, 2011

DOI: $10.3892 /$ etm.2011.367

\begin{abstract}
Glioblastoma multiforme (GBM) is the most common and lethal malignant primary brain tumor. It is classified by the World Health Organization (WHO) in the group of diffusely infiltrating astrocytomas, representing up to $50 \%$ of all primary brain gliomas, and carries the poorest prognosis. Aberrant genetic events and signaling pathways have clearly demonstrated that GBM is highly anaplastic and a morphologically highly heterogeneous tumor. Understanding the genetic alterations, specific molecular biomarkers and proliferative pathways may promote therapeutic development for the management of GBM. Age, Karnofsky performance score, histology, position and the extent of tumor resection have been identified as potential prognostic factors for patients with GBM. In this study, we review the molecular characterization of tumor cells, the current standard of care for patients diagnosed with GBM, including gross or near-total resection of the tumor, followed by radiotherapy, stereotactic brachytherapy, chemotherapy and new targeted therapies. Thus, we conclude that multimodal approaches for the treatment of patients with GBM may significantly improve their prognoses.
\end{abstract}

\section{Contents}

1. Introduction

2. Molecular characterization

3. Genetic alterations

4. Specific molecular biomarkers

5. GBM subtypes

6. Clinical manifestations

7. Current standard of care

Correspondence to: Dr Xiang Zhang, Institute of Neurosurgery, Xijing Hospital, Fourth Military Medical University, No. 127, West Changle Road, Xi'an, Shaanxi 710032, P.R. China

E-mail: xzhangns@163.com

*Contributed equally

Key words: glioblastoma multiforme, malignant glioma, genetics, molecular markers, targeted therapies
8. New targeted therapies

9. Conclusion

\section{Introduction}

Glioblastoma multiforme (GBM) is the most frequent and aggressive primary brain tumor in humans, representing up to $50 \%$ of all primary brain gliomas, and the prognosis of patients with GBM remains poor $(1,2)$. A grading scheme that has been proposed by the World Health Organization (WHO) distinguishes four different grades of gliomas. One of these, GBM WHO IV with predominant astrocytic differentiation, accounts for approximately $12-15 \%$ of all brain tumors and $60-75 \%$ of astrocytic tumors, and is the most malignant type (3). Approximately 51,000 primary brain tumors are diagnosed in the United States each year, 36\% of which are gliomas. Half of these are GBM, with approximately 3 in 100,000 individuals newly diagnosed with GBM each year (4). The treatment difficulty is due to the exceptionally infiltrative nature of GBM and its proclivity to integrate into normal brain tissue (5). Fortunately, it is worth noting that, with the notable recent advances in therapy, an increasing number of GBM patients are surviving for 36 months or longer, so that they are referred to as long-term survivors (LTS, $\geq 36$ months) (6). To date, the management of patients with GBM continues to harbor significant challenges, and comprehensive genetic screens of tumor tissues and signaling pathways have been explored to develop molecular-based targeted therapies (7).

\section{Molecular characterization}

Although numerous genetic alterations have been described in GBM $(8,9)$, such markers have proven to be of marginal utility in predicting outcome or guiding decisions regarding disease management. In general, the molecular characterization of GBM should provide a better understanding of the genomic landscape of GBMs and more efficacious means for rapid, high-throughput analyses of tumor cells and tissues.

Despite common clinical presentations and histology, it has been clearly demonstrated that GBM is a highly anaplastic and morphologically highly heterogeneous tumor. The presence of microvascular proliferation and/or necrosis is an essential 
criterion for the diagnosis of GBM (10). The diagnosis of GBM has been based on a complete clinicopathological assessment and this has been an extremely valuable approach. The pathognomonic features that characterize GBM at the tissue level are the presence of areas of necrosis with surrounding pseudopalisades and microvascular hyperplasia, which are believed to be instrumental to its accelerated growth (11).

\section{Genetic alterations}

Previous studies of the GBM genome and signaling pathways have provided a more complete view of the landscape of such alterations and their linked pathways. They indicate that genetic loss is scattered across the entire genome, affecting almost all chromosomes at frequencies ranging up to $80 \%$ of those of GBMs. The common genetic alterations include epidermal growth factor receptor (EGFR) amplification, mutations in TP53, P16, DCC and RB, and deletions associated with chromosomes $19 \mathrm{q}$ and $22 \mathrm{q}$, chromosome 7 gain and chromosome 10 loss $(12,13)$. In particular, loss of chromosome $10 \mathrm{q}$ is a more frequent occurrence in GBM than anaplastic astrocytoma and has been associated with short GBM survival (14). Albarosa et al analyzed 53 GBMs and found that the loss of heterozygosity occurred in $>90 \%$ of the tumors (15).

To identify the genetic alterations in GBMs, Parsons et al sequenced 20,661 protein coding genes, determined the presence of amplifications and deletions, and performed gene expression analyses in 22 human tumor samples. They found recurrent mutations in the active site of isocitrate dehydrogenase 1 (IDH1, an enzyme that catalyzes the oxidative carboxylation of isocitrate to $\alpha$-ketoglutarate) (16). The patients with mutated IDH1 have distinct clinical characteristics, including a considerably improved clinical prognosis. IDH1 is localized within the cytoplasm and peroxisomes and its activity leads to nicotinamide adenine dinucleotide phosphate production, and is thought to play a role in the cellular control of oxidative damage (17). Mutations in IDH1 occur predominantly in a large proportion of young patients, which is a hallmark of early cancerigenesis, and are typically associated with low-grade tumors, in accordance with the research results of Bleeker et al (18). The patients who have IDH1 mutations have a high frequency $(>70 \%)$ of TP53 mutations and a very low frequency of mutations in other commonly altered GBM genes (19). These studies support the evidence that IDH1 is a pivotal GBM cancer gene that has mutated and identify a potentially useful genetic alteration for the classification and targeted therapy of GBMs $(17,20)$. It is conceivable that new treatments may be designed to take advantage of IDH1 alterations in these patients; for example, inhibition of a different IDH enzyme (IDH2) may increase sensitivity of tumor tissues to a variety of chemotherapeutic agents (21).

Pediatric GBMs have a pattern of chromosomal and genetic modifications that are distinct from those in adults. The overexpression of the EGFR protein is observed in $23-40 \%$ of patients, and p53 gene mutations are very frequent, occurring in approximately 33-63\% of patients (22-24). The genes overexpressed in GBM usually produce extracellular proteins, thereby providing possible therapeutic targets. In addition, loss of p16 and p27 expression is observed in 68 and $54 \%$ of cases, respectively, which is similar to that observed in adult
GBMs (23). A similar study by Rood and MacDonald detected overexpression of EGFR, mutations of PTEN, deletions or epigenetic inactivation of p16, and amplification of MDM2 in pediatric GBMs (25).

\section{Specific molecular biomarkers}

With our current understanding of the expression of specific molecular biomarkers, the use of methylguanyl methyltransferase (MGMT) promoter methylation status for routine diagnostic or therapeutic purposes is considered to be a promising molecular factor that is predictive of the response of GBM patients to treatment (26). It is associated with prolonged progression-free and longer overall survival in patients with GBM who receive alkylating chemotherapy with carmustine, lomustine or temozolomide (TMZ). The MGMT gene is located on chromosome 10q26 and encodes a DNA-repair protein that removes alkyl groups from the $\mathrm{O} 6$ position of guanine, a significant site of DNA alkylation (27). For chemotherapy in patients with GBM, as a standard alkylating agent, TMZ-induced injury is repaired by the DNA repair enzymes, including MGMT, which is a unique DNA repair enzyme in the context of alkylating chemotherapy that removes the DNA methylation that is produced by TMZ. It is believed that alkylating agents cause cell death by forming cross-links between adjacent strands of DNA, owing to alkylation at the position of O6-guanine in DNA. Epigenetic silencing of this gene by promoter methylation is associated with loss of its expression and diminished DNA-repair activity. It becomes permanently inactivated and depleted in the process. Hypermethylation of the MGMT promoter decreases the expression of the protein and, as a result, DNA damage from alkylating agents is not repaired, leading to tumor cell death (27). The epigenetic silencing of the MGMT gene through promoter methylation is correlated with a median survival in patients who receive radiotherapy (RT) with TMZ for the treatment of GBM. It is now commonly recognized that silencing of the MGMT gene promoter by methylation is associated with improved GBM response to combination treatment with radiation and TMZ (28).

\section{GBM subtypes}

A number of studies have investigated molecular subclasses in GBM. Significantly, expression profiling studies have revealed that molecular classification of gliomas may be of prognostic value (29-31). From a histopathological point of view, the majority of GBMs (accounting for approximately $90 \%$ of tumors) are diagnosed as de novo or primary tumors, are more common in males and manifest a very rapid development of clinical symptoms. Secondary GBM (occurring in approximately $10 \%$ of tumors) progresses from lower-grade tumors (WHO grade II/III) with a mean progression time of approximately 55 months (32). Secondary GBM is observed in younger patients, is more evenly distributed between the genders, and exhibits longer survival times $(33,34)$. Secondary GBM may be diagnosed by clinical (neuroimaging) or histological evidence of evolution from a less malignant astrocytoma (35). Noushmehr et al have identified and characterized a distinct molecular subgroup in GBM tumors (13). Based on the context of The Cancer Genome Atlas (TCGA), they found that a distinct 
subtype of samples display concerted hypermethylation at a large number of loci, indicating the existence of a glioma-CpG island methylator phenotype (G-CIMP). G-CIMP-positive samples are associated with secondary or recurrent tumors, and are tightly associated with IDH1 somatic mutation. G-CIMP tumors also showed a relative lack of copy-number variation commonly observed in GBM. These findings identify G-CIMP as a distinct marker of human GBM using molecular and clinical features. Van Meir et al suggested a new subtype in the classification of GBM. They uncovered new genetic alterations and provided preliminary evidence that GBM may be subdivided into four subtypes (36). Verhaak et al proposed a robust gene expression-based molecular classification of GBM into proneural, neural, classical and mesenchymal subtypes, and integrated multidimensional genomic data to establish patterns of somatic mutations and DNA copy number (37).

\section{Clinical manifestations}

Clinical manifestations of GBM depend on the age of the patient, location, size and rate of growth of the tumor. Clinical manifestations include progressive headaches, dizziness, seizures, increased intracranial pressure, focal neurological deficits or changes in mental status (38). Aside from symptoms and signs, the best imaging diagnostic method is a T1-weighted gadolinium-enhanced MRI, particularly the three-planar images (Fig. 1A and B) and diffusion tensor imaging. Radiologically, GBMs present with irregular contours and a peripheral zone with strong contrast enhancement around a darker, hypodense, necrotic area and with the non-enhancing tumor extending outside the area of enhancement (39). Magnetic resonance spectroscopy identifies tumor masses with a marked increase in the choline to creatine ratio, reduction of $\mathrm{N}$-acetylaspartate and the presence of a lactate-lipid peak greatly assists neurological oncologists in diagnosis and surgery. PET and SPECT imaging may be useful tools in screening occult systemic disease. If MRI is contraindicated, a contrast-enhanced CT scan should be used. Relevant responses to treatment may be assessed by neurological examination and by MRI (Fig. 2A and B) or contrast-enhanced CT scan following therapy, or during follow-up.

With regards to gender, there is a higher incidence of GBM in males, adjusted to the World Standard Population, of 3.32 in males and 2.24 in females $(1,40)$. GBM occurs in individuals of any age, although the median age is $>60$ years, according to population-based studies. By contrast, the median age of LTS is 51 years. This is in accordance with numerous clinical studies that indicate that young age at the time of diagnosis is a significant parameter associated with LTS. At the same time, the median age in data that were collected from all 281 published GBM LTS cases during the period from 1950 to 2006 is 36.9 years. These findings underline the association of GBM LTS with prognostically favourable clinical factors, in particular young age and good initial performance score, as well as MGMT promoter hypermethylation (41). The investigators also found that primary GBMs commonly develop in older individuals (mean, 55 years), whereas secondary GBMs are found in middle-aged individuals (approximately 39 years of age) (42). In contrast to adult populations, GBM is rarely observed in children compared with adults, comprising only $5-10 \%$ of childhood intracranial neoplasms (24).

\section{Current standard of care}

The current standard of care to improve the survival of patients who have GBM begins with surgical removal as the initial treatment of choice, followed by radiation and then chemotherapy. Besides establishing a definitive histopathological diagnosis, gross or near-total resection, if feasible, may lead to rapid improvement of clinical symptoms and a reduction of steroid doses or dehydrating agents. In turn, this will dictate subsequent therapy options and significantly improve survival (43). Following surgery, a combination of RT and TMZ followed by adjuvant therapy continues to be the most effective therapy available for patients with GBM. Mineo et al showed that $80 \%$ of patients received RT prior to chemotherapy. The median survival rate was longer when RT and chemotherapy were combined versus chemotherapy alone (16 months versus 11 months) (44). Glas et al reported a survival rate of $15.4 \%$ (6/39) after 5 years. The rate of patients who are surviving long term and who are potentially cured is increased in this study, compared with the rate of 4 to $5 \%$ that is historically reported for $\operatorname{GBM}(45,46)$.

In the National Cancer Institute population databases, the five-year survival rates are approximately $13 \%$ for $15-45$ year olds, but only $1 \%$ for those aged 75 and older (39). Younger patients survive significantly longer than patients in all other age groups. The median survival ranges from 8.8 months ( $<50$ years) to 1.6 months ( $>80$ years). Liu et al compared differences in genetic variation between short-term survivors (STS, <12 months) and LTS, and explored the classification for survival data. They revealed that patients $>50$ years old with LIG4 rs7325927 had the worst survival (median survival time of 1.2 years) and exhibited the highest risk of mortality compared to younger patients with combined RTEL1 rs2297440 and HMGA2 rs1563834 genotypes (median survival time of 7.8 years) (47). Nevertheless, favorable prognostic factors were closely related to young age, good Karnofsky performance status (KPS) score, tumors located in the white matter of the frontal, temporal or occipital lobe, and no tumors in the deep structures of the brain, such as the basal ganglia, diencephalic structures or the brain stem, histology and the extent of tumor resection $(48,49)$.

Interstitial brachytherapy has been used as an adjuvant treatment for malignant brain tumors as a component of the standard therapy. In analyzing the results of this treatment, numerous publications indicate an improvement in median survival for highly selected patients. While iodine-125 (I-125) remains the most popular radionuclide for brachytherapy, there is a move away from temporary high-activity implants to permanent low-activity implants (50). This is a technique that is used to treat small $(<4 \mathrm{~cm})$, radiographically well-defined lesions with a single high-dose fraction of ionizing radiation in stereotactically directed narrow beams. This may provide added improvement to the survival outcome (51). An advantage is that brachytherapy is noninvasive, allowing treatment of patients with tumors in surgically inaccessible or eloquent areas of the brain or in those with serious coexisting medical illnesses. Stereotactic brachytherapy (SB) is used in selected patients who have supratentorial tumors that do not involve the corpus callosum, brain stem, thalamus, intraventricular or ependymal surfaces, and are unifocal, well-defined and $1-4 \mathrm{~cm}$ 

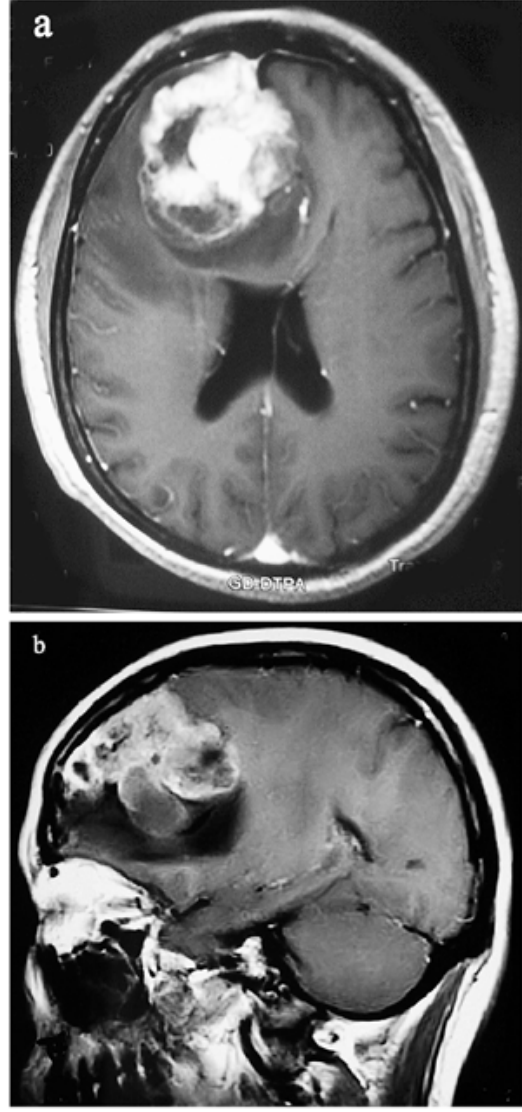

Figure 1. Imaging of a 58-year-old female who was admitted to our hospital with a 25-day history of headaches, nausea and vomiting, visual deficits and sporadic epilepsy. A nervous system examination revealed clear consciousness, but weakness and decreased visual acuity; decreased myodynamia, hyperreflexia on the left side of her body, and a positive left Babinski's sign. The KPS score was 50. Contrast-enhanced MR images: (A) axial and (B) saggital showing a large enhancing mass in the deep site of the right frontal lobe and involved to midline. Surrounding edema and the cerebral compression with midline shift are also evident. The lesion has a mixed signal with hyperintense or hypointense signaling on T1-weighted gadolinium-enhanced images. Given the signs of increased intracranial pressure and acute neurological deterioration caused by the space-occupying lesions, an instant craniotomy for tumor resection was performed and the tumor was completely removed. Histopathological examination confirmed the diagnosis of GBM.

in diameter. SB uses stereotactic techniques to accurately place inflating balloon catheters that contain radioactive isotopes or interstitial diffusion-based drug delivery systems within brain tumors, without a cytotoxic effect on normal brain structures. Typically, SB delivers an additional 50-60 Gy of radiation, bringing the total dose of radiation up to 110-120 Gy, and a mean dose of $50 \mathrm{~Gy}$. A high and heterogeneous drug concentration via chronic low-flow microinfusion (controlled-release polymer implants, i.e, Gliadel wafers) may be subtherapeutic or toxic. An intratumoral microdialysis catheter is used for a small volume to be distributed within the tumor and the brain surrounding it (52).

\section{New targeted therapies}

Although the available standard of therapy for GBM has evolved into multimodality measures, the majority of patients still experience tumor progression due to the diffuse infiltration of malignant tumor cells into the brain tissues following this treat-
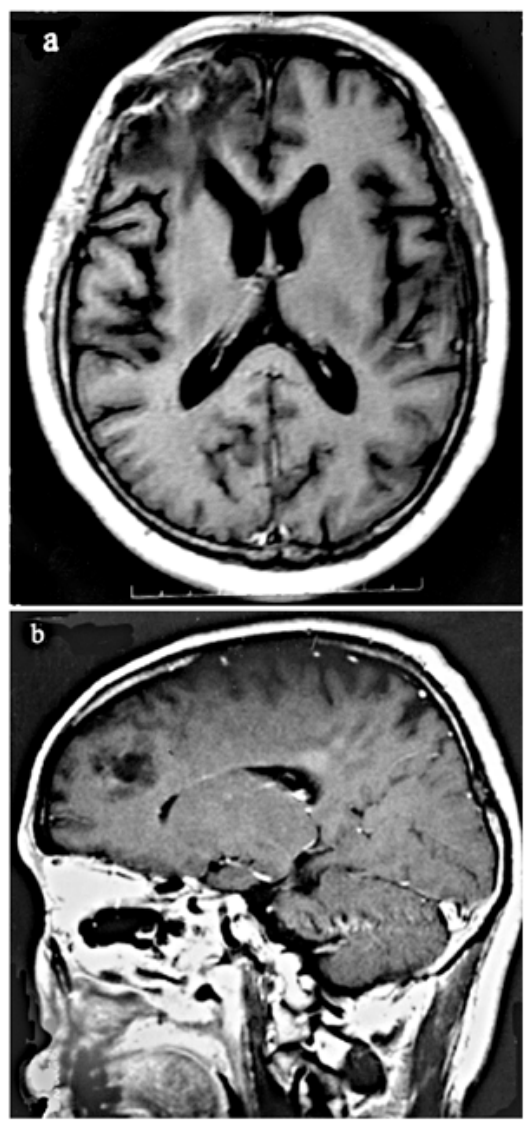

Figure 2. (A) Axial and (B) saggital T1-weighted MRI of the same female, 3 years after treatment, showing complete tumor disappearance. The patient received RT 2 weeks postoperatively, and then TMZ chemotherapy with oral administration 4 weeks postoperatively, and a maximum of three courses/year were delivered. The patient is alive and free of progression at the time of the most recent observation, 39 months following initial diagnosis.

ment. Thus, new ideas and more sensitive methods for treatment have been proposed to target therapies with the goal of increasing the specific efficacy for these patients (53). Additionally, radiation has been promoted in the form of stereotactic radiosurgery for newly diagnosed GBM or tumor recurrence.

The clinical literature confirms that GBM is a highly vascularized tumor that relies on angiogenesis, the formation of new blood vessels. The vascular structure of GBM is disorganized, tortuous and functionally abnormal, which leads to hypoxia, acidosis, increased interstitial pressure, blood brain barrier disruption and cerebral edema or tissue necrosis $(28,54)$. VEGF-A is the predominant growth factor that is expressed by GBM cells and signal transduction is mediated through its receptor, VEGFR-2, which is highly expressed in the glioma-associated endothelial cells. A number of targeted antiangiogenesis agents are currently being explored, which may normalize blood vessels and enhance delivery of oxygen and cytotoxic agents to prevent tumor progression and resistance to therapy (55). Bevacizumab (BV), a recombinant humanized IgG1 monoclonal antibody (MAb) that targets VEGF (a key regulator of tumor angiogenesis) is the first antiangiogenic therapy approved for use in cancer, and received FDA approved for the treatment of rGBM following primary therapy in 2009 (54). BV produces response rates of approximately 20 to $40 \%$ in GBM and increases 6-month progression-free survival 
(PFS6) to approximately 30 to $50 \%$ (56), which is superior to the $21 \%$ PFS6 rate reported for TMZ (57). In a study using BV monotherapy for rGBM, Moustakas et al reported that the majority of patients showed a stable performance on a variety of tests at the six-week follow-up and 18 to $25 \%$ had improved performance (58). These reports demonstrated that BV therapy leads to rapid reductions in peritumoral edema, often permitting a decrease in dose or even cessation of corticosteroid use. The MAb study proved that multivalent proteins are engineered to have high selectivity and affinity to antigenic epitopes, and are capable of functioning on the eliminable side of blood vessels without a need to traverse the blood-brain barrier. This may be effective in the treatment of brain tumors (59). Luther et al found that MAb has the potential to target GBMs. MAb-8H9 is specific for membrane protein $\mathrm{B} 7 \mathrm{H} 3$ and is reactive with the majority of human malignant gliomas. The investigators tested the $8 \mathrm{H} 9 \mathrm{scFv}-\mathrm{PE} 38$ recombinant pseudomonas immunotoxin in a preclinical model for malignant glioma. For rats harboring intracranial U87 xenografts, an infusion of $8 \mathrm{H} 9 \mathrm{scFv}-\mathrm{PE} 38$ increased the mean survival (60). Tumors also showed a volumetric response to an infusion of $8 \mathrm{H} 9 \mathrm{scFv}-\mathrm{PE} 38$ by MRI. An interstitial infusion of $8 \mathrm{H} 9 \mathrm{scFv}-\mathrm{PE} 38$ has shown potential for the treatment of hemispherical and brain stem glioma. Recently, Iwatade et al (61) evaluated the effectiveness of various anticancer drugs for MGMT-positive GBM. They found that individuals expressing MGMT-positive tumors, platinum agents and taxanes had a more significant efficacy than other categories of anticancer agents. The median survival following therapy was 20.1 months for their 74 patients with MGMT-positive GBM.

\section{Conclusion}

GBM is the most common and most malignant brain tumor in adults and carries the poorest prognosis. Recent progress in molecular biology, neuroimaging and neurosurgical care, has led to the increasing use of new targeted therapies on a multi-modality standard treatment basis in the management of GBM. The median survival time for patients with GBM has improved from an average of 12 months. The molecular-based targeted therapies being tested in clinical trials represent a new era in GBM therapeutics that bring hope to those individuals who are afflicted with this refractory disease, which may have a significant impact on quality of life for patients with GBM.

\section{Acknowledgements}

We thank Dr Junli Huo for preparing the materials of the patients, and Dr Yijun Chen for his editorial review (Stanford Comprehensive Cancer Center, Stanford University School of Medicine, Stanford, CA, USA).

\section{References}

1. Ohgaki H, Dessen P, Jourde B, et al: Genetic pathways to glioblastoma: a population-based study. Cancer Res 64: 6892-6899, 2004.

2. Stupp R, Mason WP, van den Bent MJ, et al: Radiotherapy plus concomitant and adjuvant temozolomide for glioblastoma. N Engl J Med 352: 987-996, 2005.

3. Louis DN, Ohgaki H, Wiestler OD, et al: The 2007 WHO classification of tumors of the central nervous system. Acta Neuropathol 114: 97-109, 2007.
4. CBTRUS: Statistical report: primary brain tumors in the United States, 2000-2004. Central brain tumor registry of the United States, Chicago, 2008.

5. Clarke J, Butowski N and Chang SR: Recent advances in therapy for glioblastoma. Arch Neurol 67: 279-283, 2010

6. Schmidinger M,Linzmayer L,Becherer A, et al: Psychometric-and quality-of-life assessment in long-term glioblastoma survivors. J Neurooncol 63: 55-61, 2003.

7. The Cancer Genome Atlas (TCGA) Research Network: Comprehensive genomic characterization defines human glioblastoma genes and core pathways. Nature 455: 1061-1068, 2008.

8. Von Deimling A, Louis DN and Wiestler OD: Molecular pathways in the formation of gliomas. Glia 15: 328-338, 1995.

9. Watanabe K, Tachibana O, Sata K, Yonekawa Y, Kleihues P and Ohgaki H: Overexpression of the EGF receptor and p53 mutations are mutually exclusive in the evolution of primary and secondary glioblastomas. Brain Pathol 6: 217-223, 1996.

10. Steck PA, Lin H, Langford LA, et al: Functional and molecular analyses of $10 \mathrm{q}$ deletions in human gliomas. Genes Chromosomes Cancer 24: 135-143, 1996.

11. Brat DJ and van Meir EG: Vaso-occlusive and prothrombotic mechanisms associated with tumor hypoxia, necrosis, and accelerated growth in glioblastoma. Lab Invest 84: 397-405, 2004.

12. Fujisawa H, Reis RM, Nakamura M, et al: Loss of heterozygosity on chromosome 10 is more extensive in primary (de novo) than in secondary glioblastomas. Lab Invest 80: 65-72, 2000.

13. Noushmehr H, Weisenberger DJ, Diefes K, et al: Identification of a $\mathrm{CpG}$ island methylator phenotype that defines a distinct subgroup of glioma. Cancer Cell 17: 510-522, 2010.

14. Schmidt MC, Antweiler S, Urban N, et al: Impact of genotype and morphology on the prognosis of glioblastoma. J Neuropathol Exp Neurol 61: 321-328, 2002.

15. Albarosa R, Colombo BM, Roz L, et al: Deletion mapping of gliomas suggest the presence of two small regions for candidate tumor-suppressor genes in a $17-\mathrm{cM}$ interval on chromosome $10 \mathrm{q}$. Am J Hum Genet 58: 1260-1267, 1996.

16. Parsons DW, Jones S, Zhang X, et al: An integrated genomic analysis of human glioblastoma multiforme. Science 321: 1807-1812, 2008

17. Lee SM, Koh HJ, Park DC, Song BJ, Huh TL and Park JW: Cytosolic NADP(+)-dependent isocitrate dehydrogenase status modulates oxidative damage to cells. Free Radic Biol Med 32: 1185-1196, 2002.

18. Bleeker FE, Lamba S, Leenstra S, et al: IDH1 mutations at residue p.R132 (IDH1(R132)) occur frequently in high-grade gliomas but not in other solid tumors. Hum Mutat 30: 7-11, 2009.

19. Peraud A, Watanabe K, Plate KH, Yonekawa Y, Kleihues P and Ohgaki H: p53 mutations versus EGF receptor expression in giant cell glioblastomas. J Neuropathol Exp Neurol 56: 1236-1241, 1997.

20. Rasheed BK, McLendon RE, Friedman HS, et al: Chromosome 10 deletion mapping in human gliomas: a common deletion region in 10q25. Oncogene 10: 2243-2246, 1995.

21. Kil IS, Kim SY, Lee SJ and Park JW: Small interfering RNA-mediated silencing of mitochondrial NADP ${ }^{+}$-dependent isocitrate dehydrogenase enhances the sensitivity of HeLa cells toward tumor necrosis factor-alpha and anticancer drugs. Free Radic Biol Med 43: 1197-1207, 2007.

22. Dohrmann GJ, Farwell JR and Flannery JT: Glioblastoma multiforme in children. J Neurosurg 44: 442-448, 1976.

23. Sure U, Rüedi D, Tachibana O, et al: Determination of p53 mutations, EGFR overexpression, and loss of p16 expression in pediatric glioblastomas. J Neuropathol Exp Neurol 56: 782-789, 1997.

24. Suri V, Das P, Pathak P, et al: Pediatric glioblastomas: a histopathological and molecular genetic study. Neuro Oncol 11: 274-280, 2009.

25. Rood BR and MacDonald TJ: Pediatric high-grade glioma: molecular genetic clues for innovative therapeutic approaches. J Neurooncol 75: 267-272, 2005.

26. Shah N, Lin B, Sibenaller Z, et al: Comprehensive analysis of MGMT promoter methylation: correlation with MGMT expression and clinical response in GBM. PLoS One 6: e16146, 2011.

27. Hegi ME, Diserens AC, Gorlia T, et al: MGMT gene silencing and benefit from temozolomide in glioblastoma. N Engl J Med 352: 997-1003, 2005

28. Palanichamy K, Erkkinen $\mathrm{M}$ and Chakravarti A: Predictive and prognostic markers in human glioblastomas. Curr Treat Options Oncol 7: 490-504, 2006. 
29. Prados MD and Levin V: Biology and treatment of malignant glioma. Semin Oncol 27: 1-10, 2000.

30. Freije WA, Castro-Vargas FE, Fang Z, et al: Gene expression profiling of gliomas strongly predicts survival. Cancer Res 64: 6503-6510, 2004.

31. Phillips HS, Kharbanda S, Chen R, et al: Molecular subclasses of high-grade glioma predict prognosis, delineate a pattern of disease progression, and resemble stages in neurogenesis. Cancer Cell 9: 157-173, 2006

32. Watanabe K, Sato K, Biernat W, et al: Incidence and timing of p53 mutations during astrocytoma progression in patients with multiple biopsies. Clin Cancer Res 3: 523-530, 1997.

33. Adamson C, Kanu OO, Mehta AI, et al: Glioblastoma multiforme: a review of where we have been and where we are going. Expert Opin Investig Drugs 18: 1061-1083, 2009.

34. Furnari FB, Fenton T, Bachoo RM, et al: Malignant astrocytic glioma: genetics, biology, and paths to treatment. Genes Dev 21: 2683-2710, 2007.

35. Ohgaki $\mathrm{H}$ and Kleihues P: Genetic pathways to primary and secondary glioblastoma. Am J Pathol 70: 1445-1453, 2007.

36. Van Meir EG, Hadjipanayis CG, Norden AD, Shu HK, Wen PY and Olson JJ: Exciting new advances in neuro-oncology: the avenue to a cure for malignant glioma. CA Cancer J Clin 60 $166-193,2010$

37. Verhaak RG, Hoadley KA, Purdom E, et al: Integrated genomic analysis identifies clinically relevant subtypes of glioblastoma characterized by abnormalities in PDGFRA, IDH1, EGFR and NF1. Cancer Cell 17: 98-110, 2010.

38. Grossman SA and Batara JF: Current management of glioblastoma multiforme. Semin Oncol 31: 635-644, 2004.

39. Kanu OO, Mehta A, Di C, et al: Glioblastoma multiforme: a review of therapeutic targets. Expert Opin Ther Targets 13 701-718, 2009.

40. OhgakiH: Genetic pathways to glioblastomas. Neuropathology 25: $1-7,2005$.

41. Krex D, Klink B, Hartmann C, et al: Long-term survival with glioblastoma multiforme. Brain 130: 2596-2606, 2007.

42. Xie D, Zeng YX, Wang HJ, et al: Amplification and overexpression of epidermal growth factor receptor gene in glioblastomas of Chinese patients correlates with patient's age but not with tumor's clinicopathological pathway. Acta Neuropathol 110: 481-489, 2005.

43. Lacroix M, Abi-Said D, Fourney DR, et al: A multivariate analysis of 416 patients with glioblastoma multiforme: prognosis, extent of resection, and survival. J Neurosurg 95: 190-198, 2001.

44. Mineo JF, Bordron A, Baroncini M, et al: Prognosis factors of survival time in patients with glioblastoma multiforme: a multivariate analysis of 340 patients. Acta Neurochir 149: 245-252, 2007.

45. Glas M, Happold C, Rieger J, et al: Long-term survival of patients with glioblastoma treated with radiotherapy and lomustine plus temozolomide. J Clin Oncol 27: 1257-1261, 2009.
46. McLendon RE and Halperin EC: Is the long-term survival of patients with intracranial glioblastoma multiforme overstated? Cancer 98: 1745-1748, 2003.

47. Liu YH, Shete S, Etzel CJ, et al: Polymorphisms of LIG4,BTBD2, HMGA2, and RTEL1 genes involved in the double-strand break repair pathway predict glioblastoma survival. J Clin Oncol 28 2467-2474, 2010

48. Steinbach JP, Blaicher HP, Herrlinger U, et al: Surviving glioblastoma for more than 5 years: the patient's perspective. Neurology 66: 239-242, 2006.

49. DeAngelis LM: Chemotherapy for brain tumors-a new beginning. N Engl J Med 352: 1036-1038, 2005.

50. McDermott MW, Sneed PK and Gutin PH: Interstitial brachytherapy for malignant brain tumors. Semin Surg Oncol 14: 79-87, 1998.

51. Liu BL, Cheng JX, Zhang X and Zhang Wei: Controversies concerning the application of brachytherapy in central nervous system tumors. J Cancer Res Clin Oncol 136: 173-185, 2010.

52. Brem H, Piantadosi S, Burger PC, et al: Placebo-controlled trial of safety and efficacy of intraoperative controlled delivery by biodegradable polymers of chemotherapy for recurrent gliomas. The Polymer-brain Tumor Treatment Group. Lancet 345: 1008-1012, 1995.

53. Sathornsumetee $\mathrm{S}$ and Rich JN: New treatment strategies for malignant gliomas. Expert Rev Anticancer Ther 6: 1087-1104, 2006.

54. Chi AS, Sorensen AG, Jain RK and Batchelor TT: Angiogenesis as a therapeutic target in malignant Gliomas. Oncologist 14: 621-636, 2009

55. Jain RK: A new target for tumor therapy. N Engl J Med 360: 2669-2671, 2009.

56. Wen PY: Therapy for recurrent high-grade gliomas: does continuous dose-Intense temozolomide have a role? J Clin Oncol 28: 2051-2057, 2010

57. Yung WK, Albright RE, Olson J, et al: A phase II study of temozolomide vs. procarbazine in patients with glioblastoma multiforme at first relapse. Br J Cancer 83: 588-593, 2009.

58. Moustakas A and Kreisl TN: New treatment options in the management of glioblastoma multiforme: a focus on bevacizumab. Oncol Targets Ther 3: 27-38, 2010

59. Sathornsumetee S and Rich JN: Designer therapies for glioblastoma multiforme. Ann NY Acad Sci 1142: 108-132, 2008

60. Luther N, Cheung NK, Souliopoulos EP, et al: Interstitial infusion of glioma-targeted recombinant immunotoxin $8 \mathrm{H} 9 \mathrm{scFv}-\mathrm{PE} 38$. Mol Cancer Ther 9: 1039-1046, 2010.

61. Iwadate Y, Matsutani T, Hasegawa Y, et al: Selection of chemotherapy for glioblastoma expressing $\mathrm{O}^{6}$-methyltransferase. Exp Ther Med 1: 53-57, 2010. 